\title{
Ephedra alte (Joint Pine): An Invasive, Problematic Weedy Species in Forestry and Fruit Tree Orchards in Jordan
}

\author{
Jamal R. Qasem \\ Department of Plant Protection, Faculty of Agriculture, University of Jordan, P.O. Box 13282, Amman 11942, Jordan \\ Correspondence should be addressed to Jamal R. Qasem, jrqasem@ju.edu.jo
}

Received 8 December 2011; Accepted 6 February 2012

Academic Editor: Ata Akcil

Copyright ( 2012 Jamal R. Qasem. This is an open access article distributed under the Creative Commons Attribution License, which permits unrestricted use, distribution, and reproduction in any medium, provided the original work is properly cited.

\begin{abstract}
A field survey was carried out to record plant species climbed by Ephedra alte in certain parts of Jordan during 2008-2010. Forty species of shrubs, ornamental, fruit, and forest trees belonging to 24 plant families suffered from the climbing habit of E. alte. Growth of host plants was adversely affected by E. alte growth that extended over their vegetation. In addition to its possible competition for water and nutrients, the extensive growth it forms over host species prevents photosynthesis, smothers growth and makes plants die underneath the extensive cover. However, E. alte did not climb all plant species, indicating a host preference range. Damaged fruit trees included Amygdalus communis, Citrus aurantifolia, Ficus carica, Olea europaea, Opuntia ficus-indica, and Punica granatum. Forestry species that were adversely affected included Acacia cyanophylla, Ceratonia siliqua, Crataegus azarolus, Cupressus sempervirens, Pinus halepensis, Pistacia atlantica, Pistacia palaestina, Quercus coccifera, Quercus infectoria, Retama raetam, Rhamnus palaestina, Rhus tripartita, and Zizyphus spina-christi. Woody ornamentals attacked were Ailanthus altissima, Hedera helix, Jasminum fruticans, Jasminum grandiflorum, Nerium oleander, and Pyracantha coccinea. Results indicated that E. alte is a strong competitive for light and can completely smother plants supporting its growth. A. communis, F. carica, R. palaestina, and C. azarolus were most frequently attacked.
\end{abstract}

\section{Introduction}

The Ephedraceae family consists of species with varied growth forms, habits and habitat requirements. Shrubby species that belong to this family have erect stems, decumbent plants up to $1 \mathrm{~m}$ high; scarious leaf sheath, at least of young shoots, 1-2 mm long, as long as the diameter of the subtended stem and longer than leaf rudiments. Climbing or prostrate plants are often with long lignified stems but when forming a shrub (after grazing), the scarious sheath is usually shorter than the diameter of subtended stem or leaves. Fleshy ripe fruiting bracts are red; the free part of the leaf is mostly less than $3 \mathrm{~mm}$ long and flowering branchlets are always arise from thicker stems with green photosynthetic bark $[1,2]$.

Ephedra is a distinct genus that consists of 50-65 species among which are shrubs, vines, but rarely small trees [3-5]. It is a dioecious plant, heavily branched, with very short scale leaves. It is a nonsucculent glycophyte that grows in natural habitats and is widely distributed in temperate regions in different parts of the world but usually common in dry and open habitats and in the deserts. It has been reported at elevations ranging from the near sea level (species around the Mediterranean Sea) to almost $5000 \mathrm{~m}$ (E. gerardiana in the Himalayas). Under drought, heat, and frost conditions in highlands in Asia, species have shown greater wood xeromorphy than do the lowland species [6]. Ephedra is recorded in mobile and stable sand dunes and wadis with sandy ground and is heavily consumed by camels and other grazing animals.

Ephedra is long known for its medicinal value in the Mediterranean because of the ephedrine alkaloid and other chemicals in the stems of most members of this genus $[7,8]$. Ephedrine has been long known to have contact allergenic properties and as being valuable in the treatment of 
asthma and many other complaints of the respiratory system [9]. Its naturally occurring isomer pseudoephedrine also appears as a contact sensitizer. However, recently Ephedraderived products have been found hazardous and may cause cardiac dysfunction and even death when excessively used [9]. Other chemicals isolated from the aerial parts of E. alte were vicenin II, ephedralone, p-coumaric, protocatechuic acids, herbacetin [10], ephedradine C, and hordenine [11]. However, Ephedra spp. are widely varied in alkaloid content while these chemicals are generally absent in roots, berries, and seeds of these species.

E. alte (synonym Ephedra aphylla Forssk) is one of the common species in different Middle East countries [1]. It is normally a shrub not more than $1.4 \mathrm{~m}$ in height, but when it grows with taller vegetation, such as along irrigation ditches, it may grow into those plants as a scandent liana [2]. It is found hanging from cracks in limestone cliffs or near wadis in sand and is often found growing in juniper forest with Pistacia, Opuntia, Daphne linearifolia, Artemesia, and Thymelaea hirsuta. It flowers and fruits from March to June. This species extends across the eastern Mediterranean to the Arabian Peninsula [12] and is the only Ephedra species within its range from dry to very dry habitats that may be somewhat more extensive, from cliffs, along wadis, and with phreatophytes along irrigation ditches [2]. It is able to grow in dry habitats to which few angiosperms have adapted and has been mentioned as somewhat peculiar in its growth habit. E. alte has been also mentioned as being of a high toxicity against Aedes aegypti larvae and thus may become an important plant in controlling disease-causing mosquitoes [12].

In Jordan, four Ephedra species, Ephedra alata Decne, Ephedra alte C. A. Mey, Ephedra foliate Boiss., and Ephedra transitoria Riedl., have been reported [13] to spread in natural habitats and recorded in areas in and close to the Jordan valley (tropical and subtropical), the Mediterranean, and Sahara Arabian. E. alte has been mentioned to grow in arid stony desert where average annual rainfall is between 1 and $15 \mathrm{~mm}$ [14]. Contrary to other species, E. alte is mobile and found climbing many fruit and forestry species, which resulted in severe growth damage and death of these in different regions in the country. It may be regarded as an ecologically dangerous and a threatening species to the survival of many woody species. The literature on its negative ecological impact and behavior as an agricultural pest is lacking; therefore, the following was thought important:

(i) recording species occurrence,

(ii) recording inflicted species by E. alte growth,

(iii) quantifying the effect of E. alte on fruit species,

(iv) visualizing any possible negative effects other than competition and smothering.

\section{Materials and Methods}

2.1. Study Procedure. This study aimed to survey E. alte in certain parts (covered by forestry and/or fruit trees) of Jordan at which this species was more frequently observed, record its growth status and woody plant species attacked, and accommodate its climbing habit. The survey was carried out during the period from 2008 to 2010 at which E. alte was recorded in cultivated fields, fruit tree orchards, forests, and range lands. The survey covered most regions in the central and northern parts of Jordan which located between $36^{\circ} 00^{\prime} \mathrm{E}$ longitude and $31^{\circ} 00^{\prime} \mathrm{N}$ latitude and included the central and northern Jordan Valley, Dead Sea, As-Salt, Wadi-Shu'aib, Zay, Baqqai, Nau'r, Amman, Ma'daba, Zarqa, Jerash, Irbid, and Ajloun, at which more than 150 vegetated sites were surveyed year round. The distance between sites was varied depending on the presence or absence of woody vegetation cover. At each site E. alte was checked and climbed host species were recorded. The bulk cover of vegetative mass of E. alte intermingled or laid over vegetation of other plants was subjectively rated as light, moderate, or high after being visually estimated in the field. Incidence was recorded as rare (found only on few plants in one geographical location or biogeographical region), limited (found on few plants localized in certain locations of 1 or 2 biogeographical regions), common (found on more than 10 plants in 1 or 2 biogeographical regions), or very common (found on many plants at multiple locations within more than two biogeographical regions). E. alte and plant species inflicted were all photographed.

In late 2010, additional search was carried out at which six sites in different biogeographical regions were selected to represent the total surveyed locations (Table 1). In each site, E. alte was checked on the climbed host species. Species and number of plants climbed from each in every site were recorded. Frequency of the attacked number of plants of each species and between all sites was determined and percentage of their aerial parts covered by $E$. alte vegetation was visualized within and between sites. E. alte cover observed over climbed species was estimated using a cover abundance scale $[15,16]$. Presence percentage was obtained from the number of $E$. alte plants in a specific site out of the six sites studied. Cover was estimated from estimates of $E$. alte vegetative mass projected on climbed tree as a percentage of total vegetative area of attacked species [17]. Climbed species frequency was used to detect changes in attacked number of plants in each species between different sites of different biogeographical regions. It is used to describe $E$. alte distribution on species forming plant community and often used in combination with density or cover estimates to measure trend or condition.

Notes on the vegetation type and dominating woody species in each site were also recorded.

2.2. Statistical Analysis. Data on the number of plants attacked by E. alte from each species and the percentage coverage of their aerial parts by the climber vegetation within and between the selected sites were subjected to the analysis of variance (ANOVA) and performed on species, sites, and species by sites using GLM procedure of SAS [18]. Means of percentage coverage of each species within each site were separated for significance using the $t$-test at $P \leq 0.05$ and 
TABLE 1: Representative sites description and plant number attacked by E. alte late in 2010 survey.

\begin{tabular}{|c|c|c|c|c|c|c|}
\hline No. & Site name & Biogeographical region & $\begin{array}{l}\text { Total plants climbed } \\
\text { by E. alte per site }\end{array}$ & $\begin{array}{c}\text { Estimated area } \\
\text { checked (ha) }\end{array}$ & $\begin{array}{l}\text { Approximate latitude } \\
\text { (m above sea level) }\end{array}$ & Common vegetation \\
\hline (1) & Amman & Mediterranean & 23 & 4 & 1000 & Olea europaea \\
\hline (2) & Amman & Mediterranean & 168 & 6 & 1000 & Olea europaea \\
\hline (3) & Zarqa & Mediterranean & 26 & 5 & 900 & Olea europaea \\
\hline (4) & Jerash & Mediterranean & 49 & 4 & 1100 & Citrus spp. \\
\hline (5) & $\begin{array}{l}\text { Wadi-Shu'aib upper } \\
\text { and As-Salt }\end{array}$ & $\begin{array}{l}\text { Subtropical- } \\
\text { Mediterranean }\end{array}$ & 46 & 3 & $-300-750$ & $\begin{array}{l}\text { Olea europaea, Citrus } \\
\text { spp. and almonds }\end{array}$ \\
\hline (6) & $\begin{array}{l}\text { South Shuna and } \\
\text { central Jordan Valley }\end{array}$ & Tropical & 49 & 5 & -255 & $\begin{array}{l}\text { Citrus spp. and } \\
\text { Zizyphus spina-christ } \\
\text { and mixtures }\end{array}$ \\
\hline
\end{tabular}

frequency of the attacked number of plants and coverage percentage of species attacked were calculated using the Chisquare test using Freq procedure of SAS.

\section{Results}

E. alte was found climbing/covering 40 plant species of fruit (9 species), and forest (13 species) trees, ornamentals (6 species) and shrubs (12 species) that belong to 24 plant families (Table 2). Wild and cultivated species smothered by $E$. alte included deciduous and evergreen species. Among severely affected fruit trees were A. communis, C. aurantifolia, O. europaea, O. ficus-indica, Prunus persica, and P. granatum. Peculiarly, E. alte was also found climbing other climbers including H. helix (a woody ornamental), Jasminum spp., Galium sp., Asparagus stipularis, and V. vinifera. Although E. alte attacked different species, but its growth development was substantially varied on different targets (Table 2). It formed a massive growth on A. communis, O. ficus-indica, and $R$. palaestina (Figure 1) but relatively smaller growth on C. siliqua, P. halepensis, P. granatum, and Q. coccifera. E. alte however, was destructive to A. communis, C. azarolus, $R$. palaestina and $Z$. spina-christi. It was more frequently observed on A. communis, O. ficus-indica, R. palaestina, and C. azarolus but rare on H. helix, Jasminum spp., P. coccinea, $P$. halepensis and $V$. vinifera (Table 3 ).

E. alte forms adventitious roots that enable it to climb and attach to stem and branches of host plant. These roots were found penetrating the cracked bark of old $P$. halepensis trees (Figure 2(a)). However, connection between these and internal host tissue was not observed indicating a commensalisms relationship.

Considering representative sites surveyed late in 2010, certain species were heavily attacked by $E$. alte with the highest number of smothered plants. Among all species, $A$. communis was most frequently climbed with a total number of 87 plants in all sites (Table 3 ) followed by $R$. palaestina $(67$ plants) and C. monogyna (49 plants). However, species such as A. stipularis, O. europaea, Q. coccifera. P. palaestina, and $R$. raetam showed moderate numbers of climbed plants. Other species had less than 10 plants attacked. Frequency of $E$. alte occurrence was the highest on A. communis, $R$. palaestina, and C. monogyna in different sites (Table 3). However, E. alte vegetative cover was highly varied between climbed species with P. persica, A. stipularis, Galium sp., I. viscose, and C. monogyna showing the highest coverage frequency by $E$. alte vegetation.

A. communis and $R$. palaestina were attacked in all sites (Table 4) followed by O. europaea and C. spinosa (in five sites). Differences in species number and vegetative cover frequency by $E$. alte were found between and within the searched sites (Table 4).

\section{Discussion}

Four Ephedra species have been reported to occur in Jordan $[13,19]$, and found growing in a wide range of habitats. E. alte appeared the most problematic since rapidly spread, invading and climbing both forest and fruit tree species in different locations. It grows at elevations from $255 \mathrm{~m}$ b.s.l to $1500 \mathrm{~m}$ a.s.l. and found in humid to dry and very dry regions to which few plant species have adapted. It was reported to extend across the eastern Mediterranean to the Arabian Peninsula.

Although E. alte grows separately in open lands sparsely or hardly covered by vegetation, it tends strongly to climb other plant species in its surroundings. It emerges beside other woody species (Figure 2(b)), climbs them, and rapidly forms a massive vegetative growth with long rope-like stems extending over aerial parts of other species. E. alte has been reported as being of unusual morphology among the North American and the European-Mediterranean species in having a strongly climbing liana habit [4] and a relatively unusual morphological feature of partially twining habit $[6,20]$. Thus it resembles many weed species in that its stems twining on the stem of climbed plant and its branches are extended from on, or in between those of host plants, compete for light, prevent photosynthesis, and become difficult to control by none highly selective herbicides or even through hand removal. Therefore, E. alte may be regarded as an aggressive weed that must be controlled if to avoid its damage to other species. Ephedra has been reported as a unique genus among gymnosperms in its high frequency of polyploidy found in about $65 \%$ of species studied [21], including $22 \%$ of species in which both diploid and polyploidy counts have been obtained. This, however, is 
TABle 2: Common, scientific, and family names, growth form, vegetative mass, incidence, and biogeographical regions of plant species attacked by E. alte in Jordan for the period 2008-2010.

\begin{tabular}{|c|c|c|c|c|c|c|}
\hline Common name & Scientific name & Family name & Growth status & $\begin{array}{l}\text { Vegetative mass } \\
\text { of } E \text {. alte }\end{array}$ & Incidence & Biogeographical region \\
\hline \multicolumn{7}{|c|}{ Fruit trees } \\
\hline Almond & Amygdalus communis L. & Rosaceae & $\mathrm{C}$ and $\mathrm{W}$ & High & Common & $\begin{array}{l}\text { Subtropical and } \\
\text { Mediterranean }\end{array}$ \\
\hline Mexican lime & $\begin{array}{l}\text { Citrus aurantifolia } \\
\text { (Christm.) Swingle }\end{array}$ & Rutaceae & $\mathrm{C}$ & High & Limited & Mediterranean \\
\hline Lemon & Citrus limon L. & Rutaceae & C & Moderate & Rare & $\begin{array}{l}\text { Mediterranean and } \\
\text { Subtropical }\end{array}$ \\
\hline Fig & Ficus carica L. & Moraceae & $\mathrm{C}$ & High & Limited & $\begin{array}{l}\text { Mediterranean and } \\
\text { Subtropical }\end{array}$ \\
\hline Grape & Vitis vinifera $\mathrm{L}$. & Vitaceae & $\mathrm{C}$ & Moderate & Rare & Mediterranean \\
\hline Indian fig & $\begin{array}{l}\text { Opuntia ficus-indica (L.) } \\
\text { Miller }\end{array}$ & Cactaceae & C & High & $\begin{array}{l}\text { Very } \\
\text { common }\end{array}$ & $\begin{array}{l}\text { Mediterranean, tropical, } \\
\text { and Subtropical }\end{array}$ \\
\hline Nectarine & Prunus persica L. & Rosaceae & $\mathrm{C}$ & High & Rare & Mediterranean \\
\hline Olive & Olea europaea L. & Oleaceae & $\mathrm{C}$ & High & Limited & Mediterranean \\
\hline Pomegranate & Punica granatum L. & Punicaceae & $\mathrm{C}$ & High & Limited & Subtropical \\
\hline \multicolumn{7}{|c|}{ Shrubs } \\
\hline Bedstraw & Galium sp. & Rubiaceae & $\mathrm{W}$ & High & Rare & Mediterranean \\
\hline $\begin{array}{l}\text { Fern-leaved } \\
\text { clematis }\end{array}$ & Clematis cirrhosa L. & Ranunculaceae & $\mathrm{W}$ & Moderate & Rare & Mediterranean \\
\hline Giant cane & Arundo donax L. & Gramineae & $\mathrm{W}$ & Light & Rare & Subtropical \\
\hline Grey asparagus & Asparagus stipularis Forssk. & Liliaceae & $\mathrm{W}$ & Moderate & Rare & Mediterranean \\
\hline Caper & Capparis spinosa $\mathrm{L}$. & Capparidaceae & $\mathrm{W}$ & High & Rare & Subtropical \\
\hline Indian fleabane & Pluchea indica (L.) Less. & Compositae & $\mathrm{W}$ & Moderate & Rare & Tropical and Subtropical \\
\hline Inula & Inula viscosa (L.) Aiton & Compositae & $\mathrm{W}$ & Light & Rare & Subtropical \\
\hline $\begin{array}{l}\text { Jerusalem } \\
\text { spurge }\end{array}$ & $\begin{array}{c}\text { Euphorbia hierosolymitana } \\
\text { Boiss }\end{array}$ & Euphorbiaceae & $\mathrm{W}$ & Light & Rare & Mediterranean \\
\hline Syrian mesquite & $\begin{array}{l}\text { Prosopis farcta (Banks and } \\
\text { Soland.) J. F. Macbr }\end{array}$ & Leguminosae & $\mathrm{W}$ & Moderate & Rare & Subtropical \\
\hline Sumac & Rhus coriaria L. & Anacardiaceae & $\mathrm{W}$ & High & Rare & Subtropical \\
\hline Sumac & Rhus tripartita L. & Anacardiaceae & $\mathrm{W}$ & Moderate & Limited & Subtropical \\
\hline Thorny burnet & $\begin{array}{l}\text { Sarcopoterium spinosum } \\
\text { (L.) Spach }\end{array}$ & Rosaceae & $\mathrm{W}$ & High & Rare & Mediterranean \\
\hline \multicolumn{7}{|c|}{ Ornamental shrubs and climbers } \\
\hline Common ivy & Hedera helix L. & Araliaceae & $\mathrm{C}$ & High & Rare & Mediterranean \\
\hline Heaven tree & $\begin{array}{l}\text { Ailanthus altissima (Mill.) } \\
\text { Swingle }\end{array}$ & Simaroubaceae & C & Moderate & Rare & Mediterranean \\
\hline Bush jasmine & Jasminum fruticans L. & Oleaceae & $\mathrm{C}$ & Light & Rare & Mediterranean \\
\hline Jasmine & Jasminum grandiflorum L. & & $\mathrm{C}$ & High & Rare & Mediterranean \\
\hline Oleander & Nerium oleander L. & Apocynaceae & $\mathrm{W}$ & Light & Rare & Subtropical \\
\hline Firethorn & Pyracantha coccinea Roem & Rosaceae & $\mathrm{C}$ & Light & Rare & Mediterranean \\
\hline \multicolumn{7}{|c|}{ Forest trees } \\
\hline Aleppo oak & Quercus infectoria Olivier & Fagaceae & $\mathrm{W}$ & High & Limited & Mediterranean \\
\hline Aleppo pine & Pinus halepensis Mill. & Pinaceae & $\mathrm{C}$ & Light & Rare & Mediterranean \\
\hline Carob & Ceratonia siliqua L. & Leguminosae & $\mathrm{C}$ & Moderate & Rare & Subtropical \\
\hline $\begin{array}{l}\text { Christ thorn } \\
\text { jujube }\end{array}$ & Zizyphus spina-christi L. & Rhamnaceae & $\mathrm{W}$ & High & $\begin{array}{l}\text { Very } \\
\text { common }\end{array}$ & Subtropical \\
\hline Cypress & $\begin{array}{l}\text { Cupressus sempervirens L. } \\
\text { var. horizontalis (Miller) } \\
\text { Gordon }\end{array}$ & Cupressaceae & $\mathrm{C}$ & High & Limited & Mediterranean \\
\hline Cypress & $\begin{array}{c}\text { Cupressus sempervirens L. } \\
\text { var. pyramidalis }\end{array}$ & Cupressaceae & $\mathrm{C}$ & High & Limited & Mediterranean \\
\hline
\end{tabular}


TABle 2: Continued.

\begin{tabular}{|c|c|c|c|c|c|c|}
\hline Common name & Scientific name & Family name & Growth status & $\begin{array}{c}\text { Vegetative mass } \\
\text { of E. alte }\end{array}$ & Incidence & Biogeographical region \\
\hline \multicolumn{7}{|c|}{ Forest trees } \\
\hline $\begin{array}{l}\text { Golden wreath } \\
\text { wattle }\end{array}$ & Acacia cyanophylla Lindley & Leguminosae & $\mathrm{C}$ & Moderate & Limited & Subtropical \\
\hline Hawthorn & Crataegus monogyna Jacq. & Rosaceae & $\mathrm{W}$ & High & $\begin{array}{c}\text { Very } \\
\text { common }\end{array}$ & Mediterranean \\
\hline Kermes oak & Quercus coccifera L. & Fagaceae & $\mathrm{W}$ & High & $\begin{array}{l}\text { Very } \\
\text { common }\end{array}$ & Mediterranean \\
\hline Mosphilla & Crataegus azarolus $\mathrm{L}$. & Rosaceae & W & High & $\begin{array}{l}\text { Very } \\
\text { common }\end{array}$ & Mediterranean \\
\hline $\begin{array}{l}\text { Palestine } \\
\text { buckthorn }\end{array}$ & Rhamnus palaestina Boiss & Rhamnaceae & $\mathrm{W}$ & High & $\begin{array}{l}\text { Very } \\
\text { common }\end{array}$ & Subtropical \\
\hline Terebinth & Pistacia atlantica Desf. & Anacardiaceae & $\mathrm{W}$ & High & Rare & Mediterranean \\
\hline $\begin{array}{l}\text { White weeping } \\
\text { broom }\end{array}$ & $\begin{array}{c}\text { Retama raetam (Forskal) } \\
\text { Webb and Berth }\end{array}$ & Leguminosae & $\mathrm{C}$ & High & Common & $\begin{array}{l}\text { Subtropical and } \\
\text { Mediterranean }\end{array}$ \\
\hline Wild pistachio & Pistacia palaestina Boiss & Anacardiaceae & $\mathrm{C}$ & Moderate & Common & Mediterranean \\
\hline
\end{tabular}

Rare: only on few plants in 1-2 sites of a biogeographical region.

Limited: on few plants localized in certain locations of 1 or 2 biogeographical regions.

Common: on certain plant species in > one biogeographical region.

Very common: on many plant species in different locations of different biogeographical regions.

C: cultivated, W: wild.

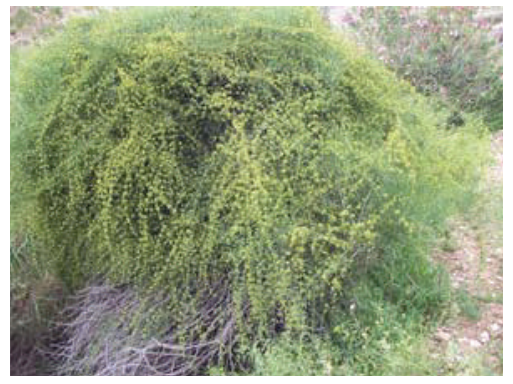

(a)

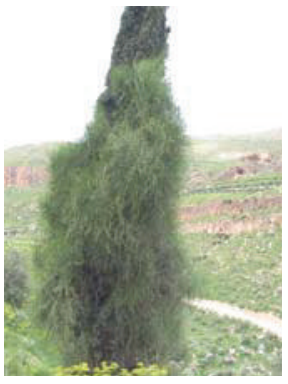

(b)

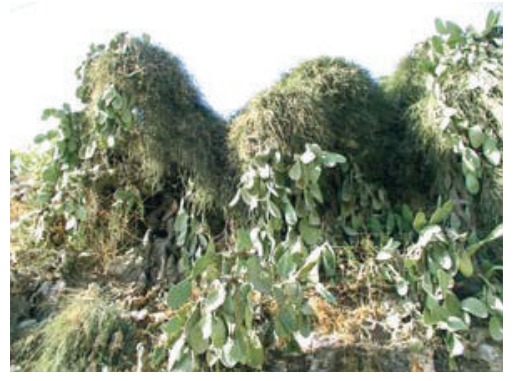

(c)

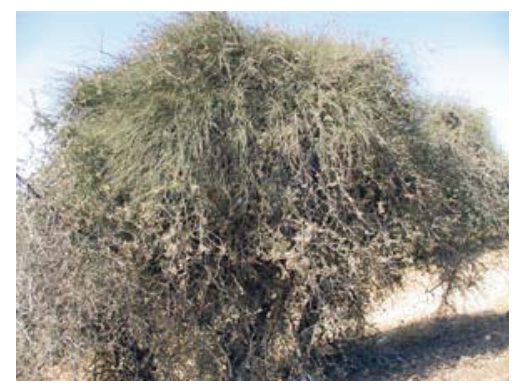

(d)

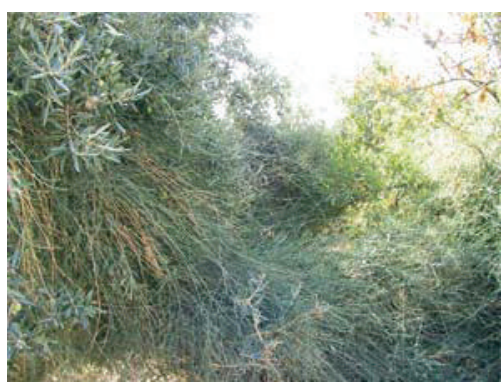

(e)

FIgURE 1: Joint-pine (a) killing Amygdalus communis, (b) climbing Cupressus sempervirens, (c) on Opuntia ficus-indica, (d) killing Rhamnus palaestina, and (e) climbing Olea europaea. Photos Scale: $23 \% \times 23 \%$.

a character shared with many noxious weed species. E. alte, however, is considered as a weed in Egypt [12].

It is well established that weeds compete for water, light, and nutrients [22]. In dense forests, light may become very limited in quantity and quality and far-reaching short stature species or low vegetation layers. It seems that $E$. alte with a unique vegetative growth (scale-like leaves, lignified stems, massive growth, etc.) tends to climb other species, forming a mattress-like vegetative cover, making it difficult for the attacked plants to perform normal photosynthesis and producing enough food to maintain growth and survive under poor fertility and low light supply. E. alte; however, it does not only prevent light from reaching effected species but its multiple stems emerge from the same point of 
TABLE 3: Total number and frequency of E. alte plants per host plant species attacked and coverage percentage on host plant in six randomly selected representative sites in Jordan late in 2010.

\begin{tabular}{|c|c|c|c|}
\hline Plant species & $\begin{array}{l}\text { Frequency of E. alte density } \\
\text { (plants per host species) }\end{array}$ & Frequency of E. alte density (\%) & E. alte coverage $(\%)$ \\
\hline $\begin{array}{l}\text { Simaroubacea } \\
\text { A. altissima }\end{array}$ & 2 & 0.55 & $77.0 \pm 15.5 \mathrm{~cd}^{*}$ \\
\hline $\begin{array}{l}\text { Rosaceae } \\
\quad \text { A. communis }\end{array}$ & 87 & 24.10 & $77.9 \pm 3.0 \mathrm{~cd}$ \\
\hline $\begin{array}{l}\text { Gramineae } \\
\text { A. donax }\end{array}$ & 1 & 0.28 & $67.0 \pm 21.5 \mathrm{bcd}$ \\
\hline $\begin{array}{l}\text { Liliaceae } \\
\qquad \text { A. stipularis }\end{array}$ & 10 & 2.77 & $88.3 \pm 7.0 \mathrm{~d}$ \\
\hline $\begin{array}{l}\text { Capparidaceae } \\
\text { C. spinosa }\end{array}$ & 8 & 2.22 & $45.5 \pm 7.7 \mathrm{ab}$ \\
\hline $\begin{array}{l}\text { Rutaceae } \\
\text { C. limon }\end{array}$ & 1 & 0.28 & $24.9 \pm 21.9 \mathrm{ab}$ \\
\hline $\begin{array}{l}\text { Cupressaceae } \\
\quad \text { C. sempervirens L. var. pyramidalis }\end{array}$ & 7 & 1.94 & $55.6 \pm 8.9 \mathrm{abc}$ \\
\hline $\begin{array}{l}\text { Rosaceae } \\
\quad \text { C. monogyna }\end{array}$ & 49 & 13.57 & $81.1 \pm 3.7 \mathrm{~cd}$ \\
\hline $\begin{array}{l}\text { Rutaceae } \\
\text { C. aurantifolia }\end{array}$ & 5 & 1.39 & $35.0 \pm 10.3 \mathrm{ab}$ \\
\hline $\begin{array}{l}\text { Euphorbiaceae } \\
\text { E. hierosolymitana }\end{array}$ & 8 & 2.22 & $71.2 \pm 7.9 \mathrm{bcd}$ \\
\hline $\begin{array}{l}\text { Moraceae } \\
\quad \text { F. carica }\end{array}$ & 5 & 1.39 & $69.9 \pm 9.8 \mathrm{bcd}$ \\
\hline $\begin{array}{l}\text { Rubiaceae } \\
\text { Galium sp. }\end{array}$ & 1 & 0.28 & $87.0 \pm 21.5 \mathrm{~cd}$ \\
\hline $\begin{array}{l}\text { Compositae } \\
\text { I. viscosa }\end{array}$ & 1 & 0.28 & $84.1 \pm 21.5 \mathrm{~cd}$ \\
\hline $\begin{array}{l}\text { Apocynaceae } \\
\text { N. oleander }\end{array}$ & 1 & 0.28 & $14.9 \pm 21.9 \mathrm{a}$ \\
\hline $\begin{array}{l}\text { Oleaceae } \\
\text { O. europaea }\end{array}$ & 19 & 5.26 & $46.8 \pm 5.2 \mathrm{ab}$ \\
\hline $\begin{array}{l}\text { Cactaceae } \\
\qquad \text { O. ficus-indica }\end{array}$ & 4 & 1.11 & $72.9 \pm 11.3 \mathrm{~cd}$ \\
\hline $\begin{array}{l}\text { Pinaceae } \\
\text { P. halepensis. }\end{array}$ & 6 & 1.66 & $52.0 \pm 8.9 \mathrm{abc}$ \\
\hline $\begin{array}{l}\text { Compositae } \\
\text { P. indica }\end{array}$ & 2 & 0.55 & $49.9 \pm 16.0 \mathrm{abc}$ \\
\hline $\begin{array}{l}\text { Rosaceae } \\
\text { S. spinosum }\end{array}$ & 5 & 1.39 & $79.2 \pm 9.8 \mathrm{~cd}$ \\
\hline $\begin{array}{l}\text { Leguminosae } \\
\text { P. farcta }\end{array}$ & 4 & 1.11 & $72.7 \pm 11.0 \mathrm{~cd}$ \\
\hline $\begin{array}{l}\text { Rosaceae } \\
\quad P \text { persica }\end{array}$ & 1 & 0.28 & $98.0 \pm 21.5 \mathrm{~d}$ \\
\hline $\begin{array}{l}\text { Anacardiaceae } \\
\text { P. palaestina }\end{array}$ & 10 & 2.77 & $56.5 \pm 7.0 \mathrm{abc}$ \\
\hline $\begin{array}{l}\text { Punicaceae } \\
\quad \text { P. granatum }\end{array}$ & 3 & 0.83 & $68.7 \pm 12.5 \mathrm{bcd}$ \\
\hline $\begin{array}{l}\text { Fagaceae } \\
\text { Q. coccifera }\end{array}$ & 15 & 4.16 & $79.4 \pm 6.4 \mathrm{~cd}$ \\
\hline $\begin{array}{l}\text { Leguminosae } \\
\quad \text { R. raetam }\end{array}$ & 26 & 7.20 & $58.9 \pm 6.7 \mathrm{abc}$ \\
\hline $\begin{array}{l}\text { Rhamnaceae } \\
\text { R. palaestina }\end{array}$ & 67 & 18.56 & $78.2 \pm 3.1 \mathrm{~cd}$ \\
\hline $\begin{array}{c}\text { Anacardiaceae } \\
\text { R. coriaria }\end{array}$ & 2 & 0.55 & $54.1 \pm 15.5 \mathrm{abc}$ \\
\hline
\end{tabular}


TABle 3: Continued.

\begin{tabular}{lccc}
\hline Plant species & $\begin{array}{c}\text { Frequency of E. alte density } \\
\text { (plants per host species) }\end{array}$ & Frequency of E. alte density (\%) & E. alte coverage (\%) \\
\hline $\begin{array}{l}\text { Anacardiaceae } \\
\quad \text { R. } \text { tripartita. }\end{array}$ & 4 & 1.11 & $47.4 \pm 12.0 \mathrm{ab}$ \\
$\begin{array}{l}\text { Rhamnaceae } \\
\text { Z. spina-christi }\end{array}$ & 7 & 1.94 & $73.5 \pm 9.8 \mathrm{~cd}$ \\
\hline
\end{tabular}

${ }^{*}$ Means within column followed by the same letter were not significantly different according to $t$-test at $P \leq 0.05$.

Numbers of $\%$ coverage represent mean values \pm SE.

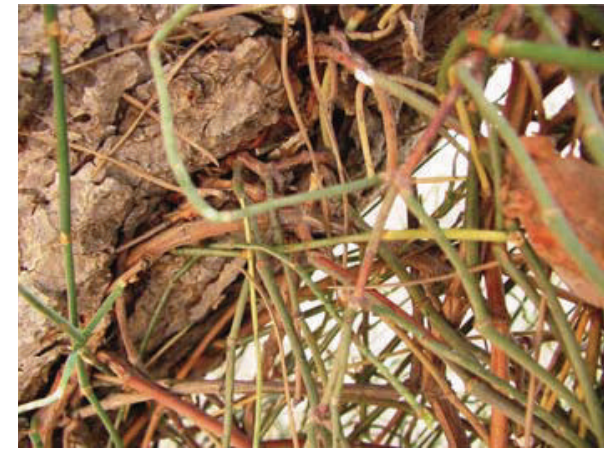

(a)

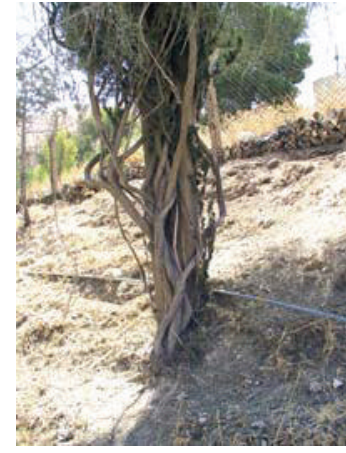

(b)

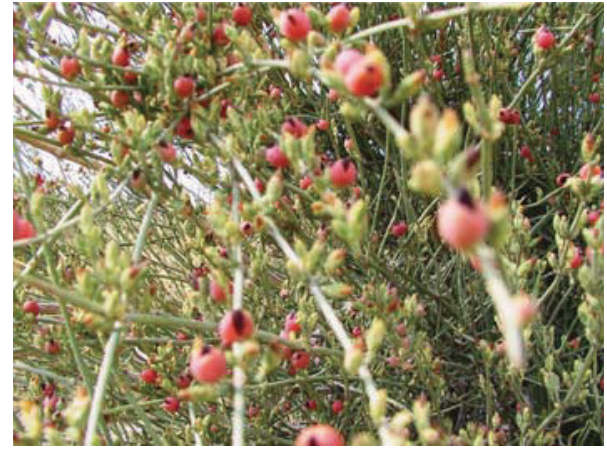

(c)

FIGURE 2: Joint-pine (a) aerial roots inserted in Pinus halepensis bark. (b) Emerged exactly with Cupressus sempervirens var. horizontalis. (c) Fruiting stage. Photos Scale: $36 \% \times 36 \%$.

host stem emergence (Figure 2(b)). This growth habit seems unusual, not fully understood [6], and somehow similar to that of certain parasitic species (e.g., Cuscuta spp.), while its tendency to grow alone as well as to climb other species is the same as that of certain hemiparasitic genera including Osyris and Thesium [23]. These parasites also grow separately, do photosynthesis, but tend strongly to parasitize other plant species. In addition, the shape, appearance, and structure of E. alte fruits more or less resemble those of some parasitic genera (Viscum, Loranthus, and Osyris). Fruit is a berry, enclosing a single seed surrounded by a sticky juicy bulb that facilitates dispersal by birds (Figure 2(c)).

Emergence of E. alte stem from the near host stem may question its self-dependence for food, nutrients, and/or water. E. alte has scale-like leaves, its photosynthate area is mainly the green stems extended over vegetative parts of host species, but photosynthetic materials produced may not be high enough to support the bulky growth it forms in densethick plant populations. Shoots of some plants of E. alte were found reddish in color which may indicate deficient mineral element/s or low chlorophyll content and thus photosynthate materials produced. However, its climbing habit frequently occurred under both dense and sparse plant populations.

Although no connection was detected between aerial parts of E. alte and attacked species, adventitious roots developed from stem nodes of this species were found inserted in the cracked bark of $P$. halepensis trees and sometimes hard to pull out from host tissues (Figure 2(a)). In addition, extension of $E$. alte stems beside host stem may suggest certain sort of connection or interrelationship between their root systems. This hypothesis, however, was not examined in the present work since $E$. alte roots were deeply extended in rocky soils. This may remain possible in form of natural root crafting which could be tested by injecting a suitable translocated herbicide into the stem of climbed species and following up any changes that may occur on growth of E. alte. It may be also examined by growing $E$. alte with a preferred and usually climbed species in a container for certain period and examining their root systems. The ambiguity in the rooting among the major groups of Ephedra is also evidenced [24] and is only likely to be solved by an examination of a number of divergent sequence regions to obtain an adequate number of informative characters. There is a limited degree of correlation between putative derived character states such as dry, winged ovulate cone bracts, single seed per cone, or unusual habit types, suggesting considerable homoplasy in the genus [6].

E. alte seems to have host preference. It climbed $C$. aurantifolia but was rarely found on Citrus limon and not recorded on Citrus orange while the surrounding C. sempervirens plants were attacked. In contrast, $E$. alte was not observed on Casuarina equisetifolia (Australian pine), Tamarix pentandra (tamarisk), or Melia azedarach (Chinaberry) trees. It attacked Z. spina-christi but not Zizyphus jujuba (common jujube). The relatively high number and diversity of targeted species may suggest certain type of association, high phenotypic plasticity, and/or physiological adaptability. Compatibility with chemicals that some of these targeted species may release into the surrounding environment is another possibility although was not tested 


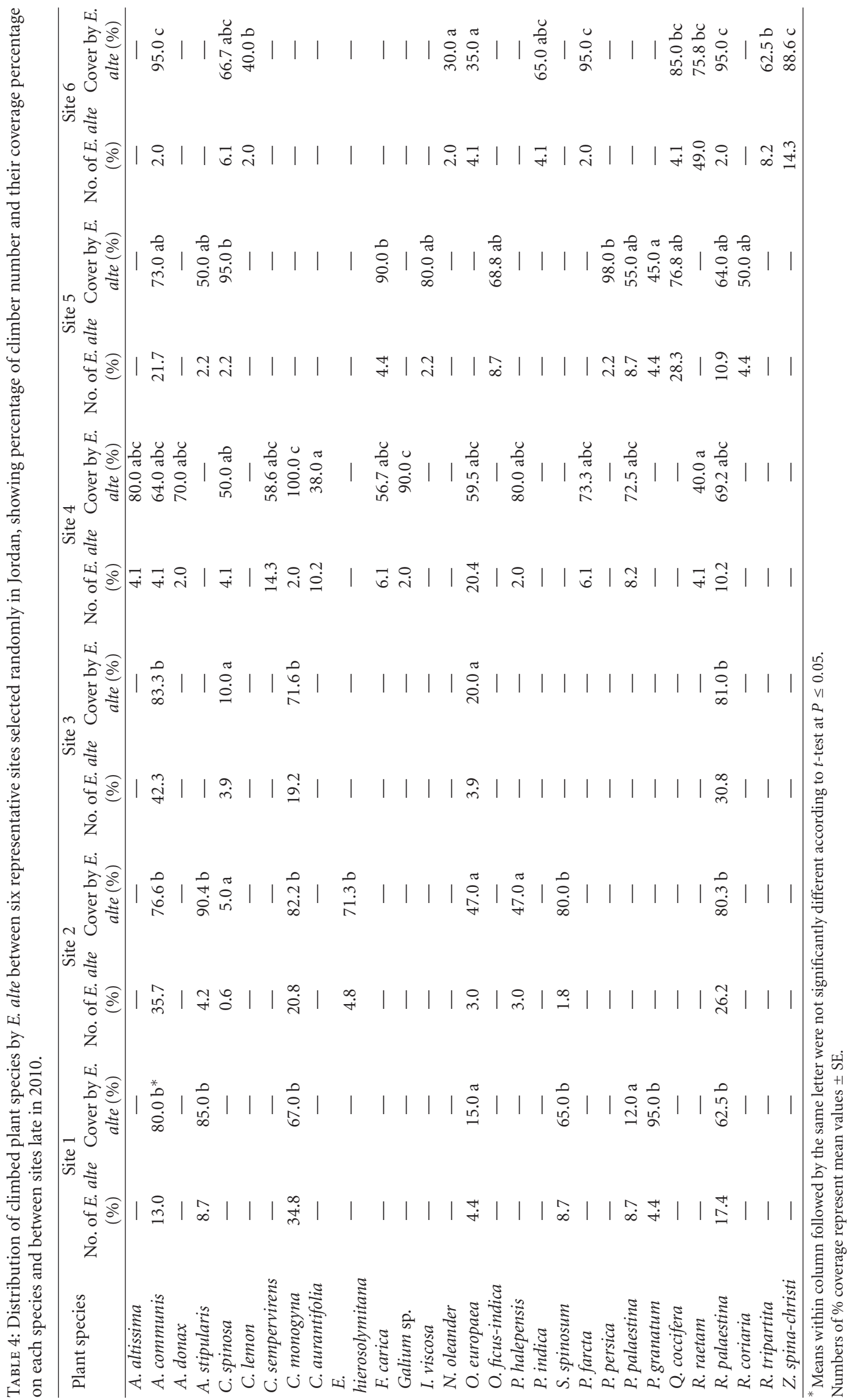


in the present work. Released chemicals may attract or repel Ephedra from climbing certain species and thus subsequently enhance or inhibit its growth. Root exudates may also have a role in whether consist beneficial/harmful compounds that stimulate/inhibit emergence and growth of $E$. alte seedlings nearby and close to these species. Root secretions may contain certain growth promoters or mineral nutrients that stimulate growth of $E$. alte and explain its association with certain woody species but not others. Although Ephedra spp. were not reported as parasites but their morphology, growth habit, and maybe responses to certain conditions are more or less similar to those of certain parasitic species. Some parasitic species are also stimulated to germinate and to grow by chemicals released from roots of their host plants. E. alte was found completely destructive to many attacked species, and its ultimate effect on host plants is more or less similar to that of different parasitic genera (Cuscuta, Loranthus, and Viscum). Moreover, similarity between E. alte and these species may be also demonstrated through the long-distance seed dispersal that is probably mediated by migratory birds [25] although the potential for overwater dispersal is also evidenced by its wide spread distribution [3].

Variations in growth of $E$. alte on different plant species may reflect differences in ecological adaptation, chemical, morphological, or physiological compatibility between these and E. alte.

Studies on Ephedra spp. control are lacking since they are not considered as weeds. However, Ephedra control may be achieved by cutting the bulky stems just emerging from the above soil level; this possibility, however, was not employed in the present work. E. alte was also found attacked by different natural enemies in Jordan including mille bugs and a scale insect from croccideae (Hemopterae) (unpublished data) which led to desiccation of its green stems. Injection of E. alte by a suitable translocated herbicide may be another control option while foliage application of herbicides can be practiced on separate Ephedra plants but not after climbing other species; otherwise low selective rates may be used or a directed treatment to avoid host injury. This, however, could be a future research line with other aspects of $E$. alte associations, host preference within and among plant species, male and female aggressiveness, and climbing habit in relation to indigenous chemicals of inflicted species. Some work on the competitive relationships between E. alte and its climbed species over nutrients and water is worth conducting.

It is worth indicating that this work is first of its kind in the country and at world level that treated E. alte as an agricultural pest of a serious threat to other woody species of different growth habit or forms. The potential harmful effect of this species on others in its vicinity was even not thought about by researchers before the conduction of this work. The present study is the first to predict possible negative association/interrelationship (through root crafting or other types) of this species with a large number of economic or ecologically important woody species in certain form of dependence on other species. In addition, E. alte is for the first time considered as a strong smothering weedy species that competes for light and maybe nutrients and/or water with host species and proved highly successful in dense as well as in sparse vegetation under different environments.

In conclusion, E. alte may be regarded as of a potential threat to different fruit and forestry species in Jordan. Its ecological harmful effects may be serious considering that forestry area represents less than $1 \%$ of the total country's area while almost $90 \%$ is a desert not receiving more than 50-70 $\mathrm{mm}$ average annual rainfall. The ecological threat this species exerts on other plant species becomes clearer when interacting with other ecological stresses, for example, poor soil fertility, frequent grazing, fire hazard, drought, and housing activities. All exert an ecological stress on the existence of certain forestry species or on the area devoted to fruit trees plantation. However, there is still much information required, and more studies are needed on E. alte prevalence in other parts of the country. Questions on climbing habit of this species in relation to ecological, physiological, and biochemical interactions with target species remained to be addressed, while its ecological threat and severity of this effect under different ecological conditions merit further research.

\section{Acknowledgments}

The author thanks the Deanship of Academic Research, University of Jordan for covering the expenses of publishing this work and Mr. M. Al-Abadi for joining in field trips.

\section{References}

[1] A. Danin and I. C. Hedge, "Contributions to the flora of Sinai-I. New and confused taxa," Notes from the Royal Botanic Garden Edinburgh, vol. 32, pp. 259-271, 1973.

[2] H. Freitag and M. Maier-Stolte, "The Ephedra-species of P. Forsskal: identity and typification," Taxon, vol. 38, no. 4, pp. 545-556, 1989.

[3] K. Kubitzki, "Gnetatae," in The Families and Genera of Vascular Plants, Pteriodophytes and Gymnosperms, K. U. Kramer and P. S. Green, Eds., vol. 1, pp. 378-391, Springer, Berlin, Germany, 1990.

[4] D. W. Stevenson, "Ephedraceae," in Flora of North America, vol. 2, pp. 428-434, Oxford University Press, New York, NY, USA, 1993.

[5] R. A. Price, "Systematics of the Gnetales: a review of morphological and molecular evidence," International Journal of Plant Sciences, vol. 157, no. 6, supplement, pp. S40-S49, 1996.

[6] J. Huang, D. E. Giannasi, and R. A. Price, "Phylogenetic relationships in Ephedra (Ephedraceae) inferred from chloroplast and nuclear DNA sequences," Molecular Phylogenetics and Evolution, vol. 35, no. 1, pp. 48-59, 2005.

[7] S. Caveney, D. A. Charlet, H. Freitag, M. Maier-Stolte, and A. N. Starratt, "New observations on the secondary chemistry of world Ephedra (Ephedraceae)," American Journal of Botany, vol. 88, no. 7, pp. 1199-1208, 2001.

[8] A. Y. Ibrahim, K. Mahmoud, and S. M. El-Hallouty, "Screening of antioxidant and cytotoxicity activities of some plant extracts from Egyptian flora," Journal of Applied Sciences Research, vol. 7, no. 7, pp. 1246-1257, 2011.

[9] R. Guharoy and J. A. Noviasky, "Time to ban ephedra-now," American Journal of Health-System Pharmacy, vol. 60, no. 15, pp. 1580-1582, 2003. 
[10] S. A. M. Hussein, H. H. Barakat, M. A. M. Nawar, and G. Willuhn, "Flavonoids from Ephedra aphylla," Phytochemistry, vol. 45, no. 7, pp. 1529-1532, 1997.

[11] M. S. Abdel-Kader, F. F. Kassem, and R. M. Abdallah, "Two alkaloids from Ephedra aphylla growing in Egypt," Natural Product Sciences, vol. 9, no. 2, pp. 52-55, 2003.

[12] http://threatenedplants.myspecies.info/sites/threatenedplants .myspecies.info/files/Species\%20Summary\%20-\%20Ephedra $\% 20$ aphylla.pdf.

[13] G. E. Post and J. E. Dinsmore, Flora of Syria, Palestine and Sinai, AUB, Beirut, Lebanon, 2nd edition, 1932.

[14] F. B. Pyatt, G. Gilmore, J. P. Grattan, C. O. Hunt, and S. McLaren, "An imperial legacy? An exploration of the environmental impact of ancient metal mining and smelting in southern Jordan," Journal of Archaeological Science, vol. 27, no. 9, pp. 771-778, 2000.

[15] J. Braun-Blanquet, Plant Sociology, McGraw-Hill, New York, NY, USA, 1932, Edited by G. D. Fuller and H. S. Conard.

[16] J. Braun-Blanquet, Pflanzensoziologie, Springer, Berlin, Germany, 3rd edition, 1964.

[17] D. Mueller-Dombois and H. Ellenberg, Aims and Methods of Vegetation Ecology, John Wiley and Sons, New York, NY, USA, 1st edition, 1974.

[18] SAS, SAS User's Guide: Statistics, SAS Institute, Cary, NC, USA, 1996.

[19] L. J. Musselman, "Checklist of plants of the Hashemite Kingdom of Jordan," 1998, Old Dominion University, http://www .odu.edu/ lmusselm/plant/jordan/index.php.

[20] I. F. Mussayev, "On geography and phylogeny of some representatives of the genus Ephedra L," Botanicheskii Zhurnal SSSR, vol. 63, pp. 523-543, 1978.

[21] J. Huang, Molecular systematics and evolution of the genus Ephedra, Ph.D. dissertation, University of Georgia, Athens, Ga, USA, 2000.

[22] R. L. Zimdahl, Weed Crop Competition: A Review, International Plant Protection Center. Oregon State University, Corvallis, Ore, USA, 1980.

[23] J. R. Qasem, "Recent advances in parasitic weed research, an overview," in Weed Management Handbook, H. P. Singh, D. R. Patish, and R. K. Kohli, Eds., pp. 627-728, The Haworth Press, Binghamton, NY, USA, 2006.

[24] D. E. Soltis and P. S. Soltis, "Choosing an approach and an appropriate gene for phylogenetic analysis," in Molecular Systematics of Plants II: DNA Sequencing, D. E. Soltis, P. S. Soltis, and J. J. Doyle, Eds., pp. 1-42, Kluwer Academic Publishers, Dordrecht, The Netherland, 1998.

[25] R. F. Thorne, "Phytogeography," in Flora of North America, vol. 1, pp. 132-153, Oxford University Press, New York, NY, USA, 1993. 

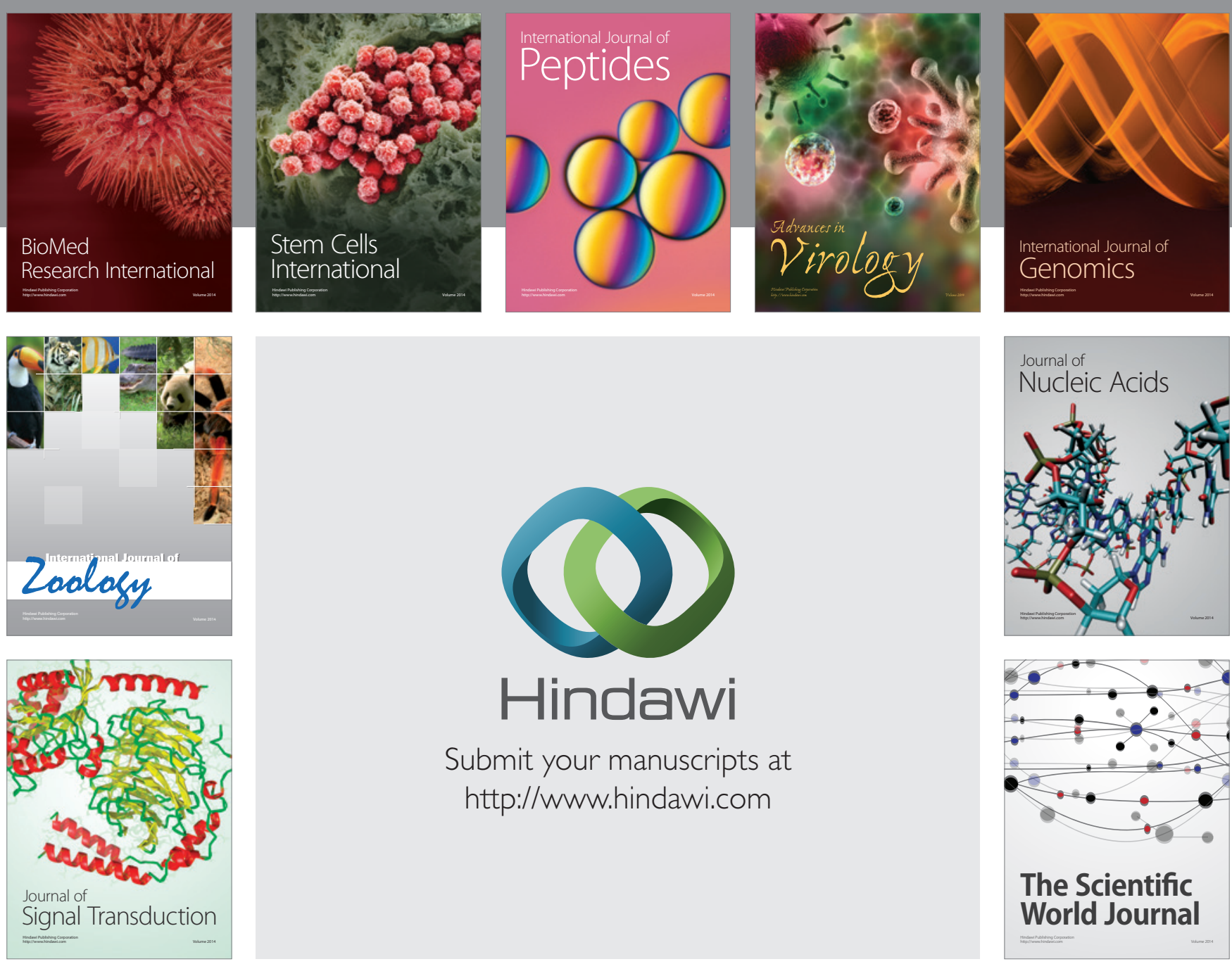

Submit your manuscripts at

http://www.hindawi.com
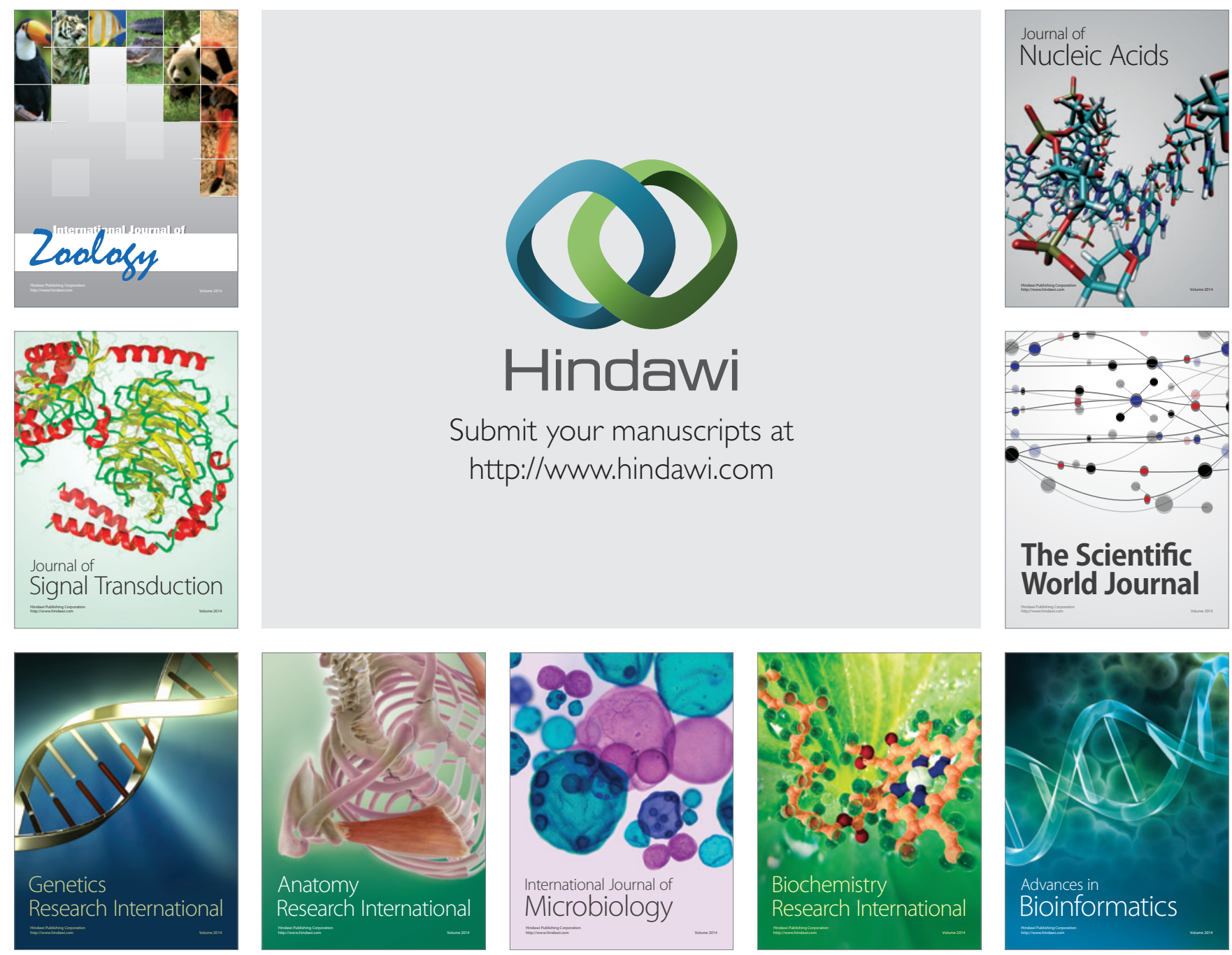

The Scientific World Journal
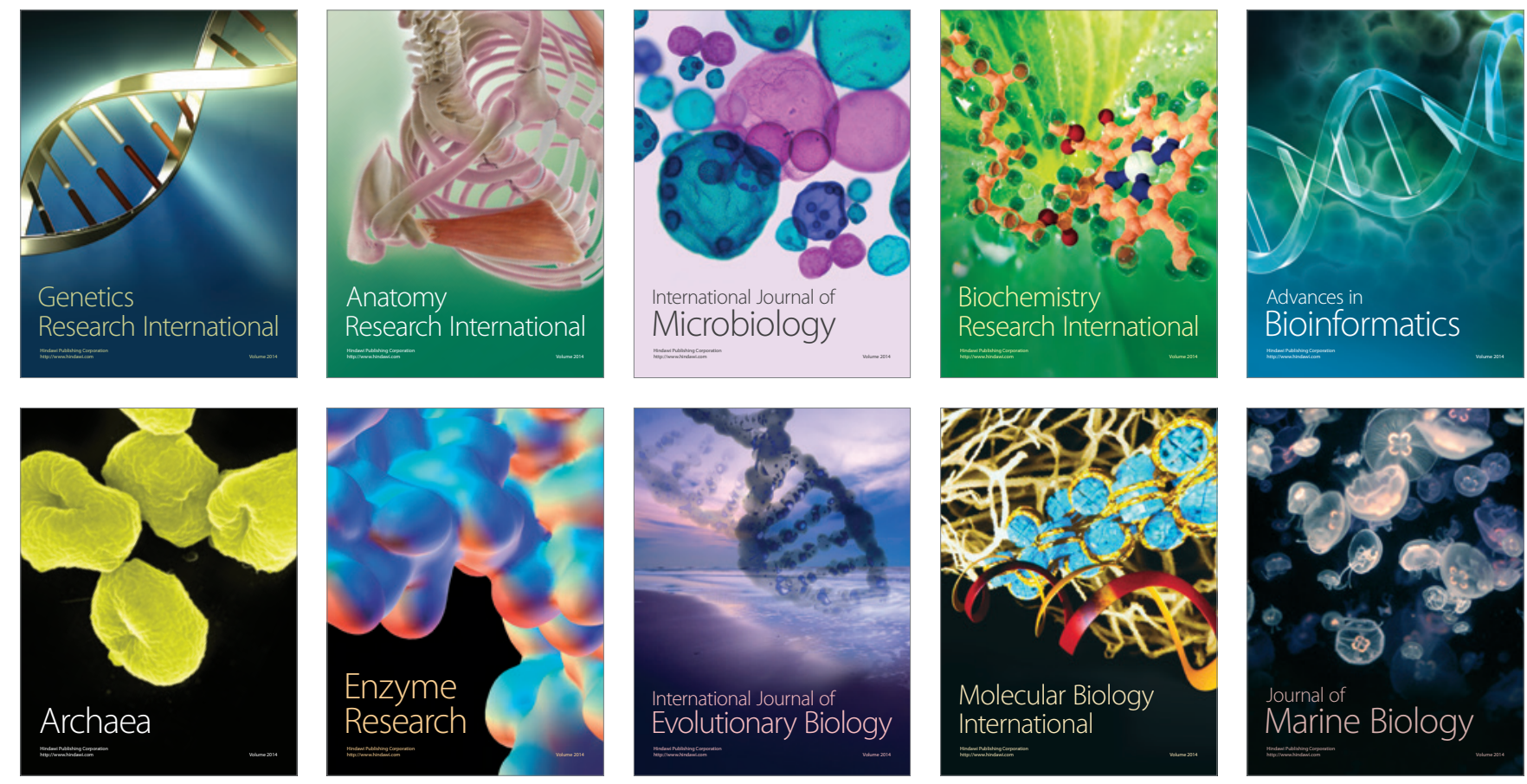